\title{
CONOCIMIENTO POPULAR Y PERSPECTIVAS DE CONSERVACIÓN SOBRE LAS TORTUGAS CONTINENTALES EN LA PARTE BAJA DEL RÍO LA MIEL (COLOMBIA)
}

\author{
Jennifer Zapata-Ciro ${ }^{1}$ \\ Giovany Guevara ${ }^{2}$ \\ Gabriel Jaime Castaño-Villa ${ }^{3}$
}

Recibido el 5 de marzo de 2015, aprobado el 6 de julio de 2015 y actualizado el 11 de mayo de 2016

DOI: 10.17151/luaz.2016.43.2

\section{RESUMEN}

La parte baja de la cuenca del río La Miel está ubicada al nororiente del departamento de Caldas y alberga cinco de las especies de tortugas continentales registradas para Colombia. Durante mayo a septiembre de 2014 se recopiló información mediante la aplicación de un cuestionario semiestructurado a 101 residentes de la cuenca hidrográfica, acerca del conocimiento y uso de las tortugas (alimento, comercio y mascota) en la zona. La encuesta incluyó información personal de acuerdo con el sexo, ocupación principal (ama de casa, pescador/lanchero, agricultor/jornalero, mayordomo), tipo de habitante (nativos, extranjeros) y conocimiento y uso de las tortugas. Los resultados sugieren que las tortugas de agua dulce en la zona de estudio, se han reducido debido a una aparente larga historia de utilización por el hombre; principalmente para alimento, uso como mascotas y ocasionalmente por el comercio. Sin embargo, estos datos también sugieren que a pesar del impacto humano el río La Miel podría concentrar un número alto de tortugas a lo largo de la cuenca, lo que resalta la importancia de este sistema acuático como uno de los hábitats más importantes para la conservación de las tortugas de agua dulce en el país. Asimismo, los resultados muestran algunas relaciones relevantes hombre-fauna silvestre, que pueden ser utilizadas para avanzar en nuevos esfuerzos hacia la gestión de la conservación de las tortugas de agua dulce bajo un contexto local y nacional.

\section{PALABRAS CLAVE}

Podocnemis lewyana, usos tradicionales de las tortugas, tortugas continentales. 


\title{
POPULAR KNOWLEDGE AND CONSERVATION PERSPECTIVES ON THE INLAND TURTLES AT THE LOWER PART OF LA MIEL RIVER (COLOMBIA)
}

\begin{abstract}
The lower part of La Miel River, located at the north-east of the Department of Caldas, hosts five Colombian inland turtle species. Information about knowledge and turtles' use (for food, trade, or pets) was recorded using a semi-structured questionnaire applied to 101 watershed residents, from May to September 2014. The survey included personal, knowledge, and use information accordingly to sex, principal work (housewife, fisherman/boatman, farmer/laborer, steward) and inhabitant type (native, foreign). Our results suggest that freshwater turtles in the study area have declined due an apparent long history of human consumption; mainly for food, pet, and occasionally for trade. However, these data also suggest that despite human impact, La Miel River could support a large number of turtles along the basin, underscoring the importance of this aquatic system as one of the most important habitats for the conservation of freshwater turtles in the country. In addition, our results illustrate some relevant human-wildlife relationships, which can be used to advance new efforts toward freshwater turtle management under local and national context.
\end{abstract}

\section{KEY WORDS}

Podocnemis lewyana, Inland turtles, traditional uses of turtles.

\section{INTRODUCCIÓN}

Colombia ocupa el séptimo lugar a nivel mundial y el segundo en Sudamérica con mayor riqueza de especies y subespecies de tortugas continentales (dulceacuícolas y terrestres) después de Brasil, además es el país con mayor número de familias (9) (Rueda-Almonacid et al., 2007; Páez et al., 2012; Van Dijk et al., 2014). Doce de las 27 especies registradas en Colombia (44\%), se encuentran amenazadas y listadas por la IUCN (2014) en alguna categoría de riesgo, debido principalmente a la pérdida y degradación de su hábitat y a su extracción masiva para consumo y comercialización (Páez et al., 2012). Las tortugas continentales, en general, juegan un papel importante en los ecosistemas fluviales y en la sociología y economía de la cultura humana (Moll y Moll, 2004). 
Dentro de las comunidades biológicas son de gran importancia, ya que participan en las cadenas tróficas, interactúan con vertebrados e invertebrados, y algunas especies son dispersoras de semillas (Páez et al., 2012). Desde lo social, las tortugas son referentes culturales y componentes importantes en la economía de subsistencia y alimentación en diversas regiones del país (Castaño-Mora, 2002).

Las tortugas continentales son uno de los taxones de vertebrados más amenazados del mundo, con más de la mitad de las especies en vía de extinción, y han sido poco estudiadas en cuanto a su distribución espacial y ecología debido probablemente al carácter críptico de muchas especies (Van Dijk et al., 2014). En Colombia, las principales amenazas directas que afectan las poblaciones de tortugas continentales son la sobreexplotación para consumo, comercio y la extracción para venta como mascotas (Páez et al., 2012). El aprovechamiento de las tortugas se da principalmente en regiones rurales y marginales del país, donde las comunidades locales las utilizan como un recurso cinegético de subsistencia y comercio (Racero-Casarrubia et al., 2008; Morales-Betancourt et al., 2012b; Castro et al., 2013; Arroyave et al., 2014).

Actualmente se considera que el éxito de los programas de conservación de la fauna silvestre, depende de la integración de aspectos biológicos, sociales y culturales (Bath, 1996; Primack et al., 2001). Por lo tanto, evaluar el conocimiento popular que las comunidades locales tienen acerca de la fauna de su entorno, contribuye al conocimiento sobre las interacciones hombre-fauna (Ojasti y Dallmeier, 2000). Lo anterior, es parte fundamental de las investigaciones aplicadas al manejo de vida silvestre, ya que permite la construcción de soluciones a los problemas ambientales locales (Valdelomar et al., 2012).

Aunque en la cuenca baja del río La Miel se han registrado cinco especies de tortugas continentales (Páez et al., 2012): Cryptochelys leucostoma (Duméril \& Bibron, 1851),Rhinoclemmys melanosterna (Gray, 1861), Trachemys callirostris (Gray, 1855),Podocnemis lewyana (Duméril, 1852) y Chelonoidis carbonaria (Spix, 1824), se desconocen las relaciones de las comunidades locales con estos organismos. En particular, no se evidencia claramente el conocimiento y uso en general de estas especies por parte de los pobladores de la cuenca, lo cual influye sobre su conservación. Lo que cobra relevancia, debido a que $\boldsymbol{P}$. lewyana y $\boldsymbol{C}$. carbonaria se encuentran dentro de las categorías de conservación global y nacional (IUCN, 2014). Por un lado, la tortuga del río Magdalena ( $\boldsymbol{P}$. lewyana) es considerada globalmente como una especie en peligro de extinción 
(Van Dijk et al., 2014), y por otro el morrocoy terrestre (C. carbonaria) es considerada en peligro crítico a nivel nacional (Castaño-Mora, 2002).

El objetivo de este trabajo fue evaluar el conocimiento popular que las comunidades locales poseen acerca de las tortugas continentales en la parte baja de la cuenca del río La Miel, y discutir algunas de las perspectivas de conservación que se puedan promover en dicha cuenca.

\section{MÉTODOS}

\section{Área de estudio}

La parte baja de la cuenca del río La Miel, un afluente del río Magdalena, está localizada en la vertiente oriental de la Cordillera Central de Colombia. La temperatura promedio es de $29^{\circ} \mathrm{C}$, con un régimen de lluvias bimodal, una precipitación media anual entre 2000-4000 mm, con periodos de lluvias entre abril y mayo, y de septiembre a diciembre, al igual que dos períodos secos: eneromarzo y junio-agosto (Vallejo-Joyas et al., 2005; Cantillo-H. y Rangel-Ch., 2011). El área seleccionada para el estudio, incluyó parte del municipio de Norcasia (Caldas), las veredas La Habana, La Atarraya y el corregimiento de Buenavista en el municipio de La Dorada (Caldas) y, el corregimiento de San Miguel en el municipio de Sonsón (Antioquia).

\section{Conocimiento y uso de las tortugas}

Se realizó un cuestionario semiestructurado a los habitantes de la región (Tabla 1). Las encuestas fueron aplicadas entre mayo y septiembre de 2014, con el objetivo de establecer el conocimiento acerca de la distribución, hábitat, alimentación, reproducción de las tortugas; así como su uso, bajo tres criterios (consumo, comercio y mascotas). Se aplicaron 101 encuestas en cuatro puntos considerados "estratégicos" (asentamientos humanos con mayor influencia o dependencia económica del río La Miel). Los lugares seleccionados incluyeron personas de La Habana $(n=17)$, Norcasia $(n=27)$, La Atarraya $(n=16)$, Buenavista $(n=21)$ y San Miguel $(n=20)$. A los participantes, se les mostraron imágenes de las tortugas reportadas para el lugar, antes de resolver las preguntas, con la intención de evitar sesgos en las respuestas relacionadas con el nombre común y rasgos descriptivos de las especies. Los aspectos relacionados con la biología, distribución y 
amenazas de estas especies, fueron contrastados y complementados con la información documentada para el país por Rueda-Almonacid et al. (2007) y Paéz et al. (2012).

Tabla 1. Cuestionario semiestructurado dirigido a los habitantes de la parte baja de la cuenca del río La Miel (Buenavista, San Miguel, La Atarraya, La Habana y Norcasia)

\begin{tabular}{|c|c|}
\hline Enfoque & Información/Pregunta \\
\hline $\begin{array}{l}\text { Información } \\
\text { personal }\end{array}$ & $\begin{array}{l}\text { - Nombre, sexo, edad, oficio, lugar de nacimiento, tiempo y sitio de } \\
\text { residencia en la zona de estudio. }\end{array}$ \\
\hline Conocimiento & $\begin{array}{l}\text { - ¿Cuántas especies (diferentes) de tortugas conoce en la región? } \\
\text { - ¿Cómo las diferencia? } \\
\text { actualmente observa con menor frecuencia? } \\
\text { - ¿Cuál o cuáles de las especies de tortugas que usted conoce en la } \\
\text { región podrían haber reducido su número de individuos? } \\
\text { - ¿Cuáles podrían ser las causas de dicha reducción? } \\
\text { - ¿Ha recibido o conoce de alguna campaña educativa o cultural que } \\
\text { trate temáticas relacionadas con las tortugas? } \\
\text { - ¿Cuál considera usted que es el papel de las tortugas dentro de la } \\
\text { naturaleza? }\end{array}$ \\
\hline Uso & $\begin{array}{l}\text { - ¿Cuáles son los usos que se les da a las tortugas en la zona? } \\
\text { - ¿Por qué y en qué épocas son usadas las especies de tortugas que } \\
\text { usted conoce? }\end{array}$ \\
\hline
\end{tabular}

\section{Análisis de datos}

Se cuantificaron las variables extraídas de las encuestas para obtener porcentajes y frecuencia de cada tipo de respuesta. Se establecieron diferencias en el conocimiento/uso de las tortugas en relación con el sexo, edad (adultos jóvenes: 18-35, adultos: 36-65 y adultos mayores: > 65 años), estatus de residencia (nativo o inmigrante con menos de 5 años en la región), oficio principal (ama de casa, pescador/lanchero, agricultor/jornalero, y mayordomo) y localidad (corregimiento de residencia) de los encuestados, por medio de la Prueba Exacta de Fisher. Los análisis estadísticos fueron realizados en R versión 3.1.1 (R Development Core Team, 2011). 


\section{RESULTADOS}

El $75 \%$ de los habitantes encuestados reconocieron cuatro de las cinco especies de tortugas registradas en el área de estudio. En su mayoría estas personas identifican estos organismos a partir de características morfológicas (e.g., la forma del caparazón y el color de la cabeza); sin embargo, existen otras personas que las asocian con su hábitat. El morrocoy terrestre solo fue reconocido por el 14\% de los encuestados. Con respecto al conocimiento sobre el uso de las tortugas para consumo, se encontró que no hubo diferencias entre sexos (Prueba Exacta de Fisher, $\mathrm{P}=0,070$ ) o la edad de los encuestados (Prueba Exacta de Fisher, $\mathrm{P}=$ 0,789 ) (Tabla 2). En general, las personas encuestadas mencionan que la especie de mayor consumo es $\boldsymbol{P}$. lewyana (86\%), de la que se aprovecha tanto la carne como los huevos; las personas describen que esta especie, es consumida por su aporte proteico, buen sabor y valor afrodisíaco. El consumo de esta especie se realiza durante todo el año, siendo el consumo de huevos solo durante los períodos de verano (enero y junio, principalmente). Por otra parte, el 33\% de los encuestados reconocen que $\boldsymbol{R}$. melanosterna, $\boldsymbol{T}$. callirostris y $\boldsymbol{C}$. carbonaria son las tres especies que se utilizan como mascota en sus hogares.

La hicotea y el morrocoy de tierra lo usan mucho para mascotas, la que si no falta en el plato es la tortuga de río, sabe muy bueno. (Pescador, Norcasia - Caldas)

Cuando se consultó acerca de la abundancia de las especies, muchos de los encuestados reconocen que en la actualidad la especie que observan con mayor frecuencia es $\boldsymbol{P}$. lewyana, pero que su abundancia ha disminuido en los últimos 15 años. Al respecto, algunos habitantes explicaron que la disminución en la abundancia de las tortugas, puede estar asociada con la destrucción de los ecosistemas, debido principalmente a la minería (pérdida del hábitat y contaminación del agua), a la construcción del Embalse Amaní (el cual alteró la dinámica de los recursos hídricos) y a la perturbación de los humedales, debido a la ganadería de búfalos (11\% de los encuestados).

Hubo un tiempo, hace como 15 años antes de que metieran las búfalas y la construcción de la presa, que sí se veían muchas tortugas, ahora no se ven tantas. (Mayordomo, Norcasia - Caldas)

El conocimiento acerca del uso de las tortugas fue significativamente diferente en relación con el oficio de los encuestados (Prueba Exacta de Fisher, P = 0,009), 
siendo los agricultores/jornaleros y los mayordomos las personas que menos reconocen los usos que se les da a las tortugas. Asimismo, se detectaron diferencias con respecto a las localidades (Prueba Exacta de Fisher, $P<0,001$ ); en particular, en La Habana y Norcasia es donde los encuestados conocen muy poco acerca de los usos que se les da a las tortugas (Tabla 2).

Tabla 2. Porcentaje de entrevistados según sexo, edad, ocupación, lugar de residencia y tiempo de residencia que mencionaron los diferentes usos que tienen las tortugas en Buenavista, San Miguel, La Atarraya, La Habana y Norcasia

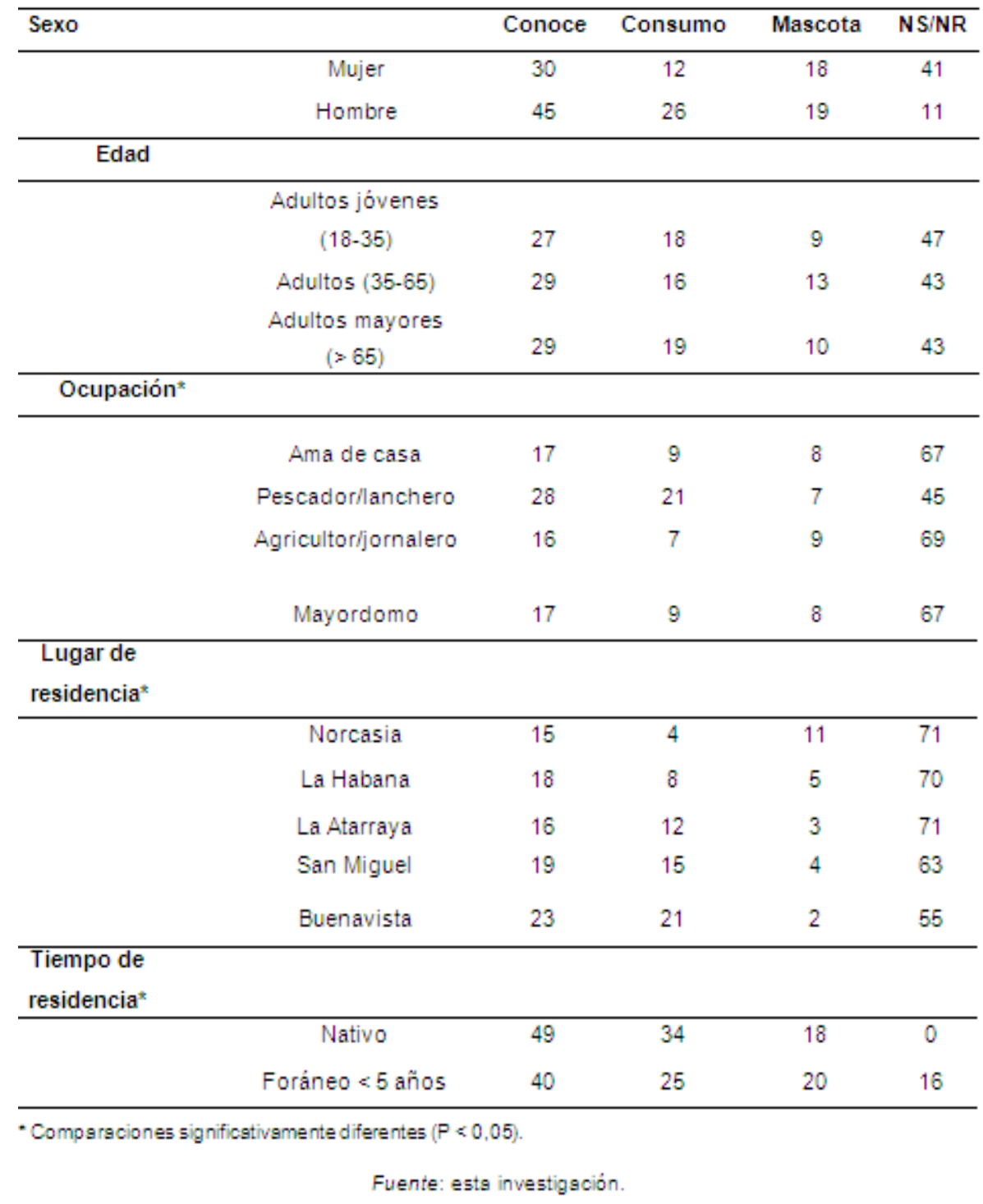

El 82\% de los encuestados no conoce el papel o importancia de las tortugas dentro de los ecosistemas, el 18\% restante las asocia con la presencia de babillas y con 
los niveles altos de agua. Aquellos con menos de 5 años de residencia en la región, son los que tienen un escaso conocimiento acerca de los usos que se les da a las tortugas, en comparación con las personas originarias de la zona (Prueba Exacta de Fisher, $\mathrm{P}<0,013)$. El 5\% de los encuestados confirmó haber recibido información relacionada con temáticas en las que se involucra la conservación de las tortugas. Esta información la han recibido solamente de CORPOCALDAS, en campañas de conservación de la tortuga morrocoy (Chelonoidis carbonaria) en el corregimiento de Buenavista (La Dorada - Caldas).

Nosotros sí recibimos campañas de educación, pero más que todo enfocada a los peces, de tortugas nunca. (Pescador, Buenavista, La Dorada - Caldas)

\section{DISCUSIÓN}

Las especies de tortugas de la región son reconocidas, en una proporción moderada, por parte de los habitantes de la parte baja de la cuenca del río La Miel. En general, el conocimiento de los habitantes se concentra en aquellas especies que se utilizan principalmente para el consumo y comercio (e.g., P. lewyana). Esto se reflejó en una mayor comprensión acerca del uso de las tortugas por parte de los habitantes de las veredas La Atarraya y Buenavista, donde se ubican playas principales de anidación y se puede observar la cacería para consumo y comercio. Por otro lado, el conocimiento también se asocia con la ocupación de los encuestados, ya que las personas que dependen del río para su subsistencia, son las que mejor conocen a las tortugas.

En parcial concordancia con investigaciones realizadas en otras regiones de Colombia (Racero-Casarrubia et al., 2008; Castro et al., 2013), los encuestados consideraron que las poblaciones de tortugas han disminuido principalmente como resultado de la intervención antropogénica, que en el caso del río La Miel ha ocasionado cambios en su dinámica fluvial, como resultado de las modificaciones ocurridas durante y después de la construcción del Embalse Amaní, a la contaminación de los recursos hídricos (minería), y a la incorporación de los humedales en actividades ganaderas (principalmente con búfalos, Bubalus bubalis). Sin embargo, contrario a la percepción en otras regiones del país, los habitantes de la parte baja de la cuenca, no asocian la disminución que ellos describen sobre las poblaciones de tortugas a la cacería, aun siendo conscientes 
que $\mathrm{P}$. lewyana es la especie con mayor demanda para el consumo regional. Lo anterior puede ser un factor clave para considerar en programas dirigidos a la protección, conservación y uso sustentable de esta especie.

En el área de estudio se pudo observar la extracción de adultos de diversas tallas (Obs. Pers. de los autores) y de los huevos de la tortuga de río (registro anecdótico, Buenavista, La Dorada - Caldas). Aunque no se cuenta con datos acerca de la intensidad y frecuencia con la que se presenta la colecta de esta especie en el área de estudio, los resultados de las encuestas mostraron una tendencia clara sobre el uso y preferencia de los habitantes de la región por su consumo. La disminución percibida por los habitantes de la región se asocia a factores como la cacería y daños ambientales, efectos que podrían alterar la composición y estructura de la comunidad de tortugas continentales, y pueden llevar a la extinción local de las especies amenazadas (Bennett y Robinson, 2000).

Varios de los factores anteriormente mencionados deben ser considerados de especial interés para la conservación de la tortuga de río en esta región. Actualmente, en Colombia, todas las especies del género Podocnemis están amenazadas, debido principalmente a la sobreexplotación (Rueda-Almonacid et al., 2007; Páez et al., 2012). Esta es una de las especies de tortuga que es cazada para consumo y comercio a través de todo su rango de distribución en el país (Castro-Herrera, 2002). Por lo tanto, es una especie de alta prioridad para la implementación de medidas de conservación, entre ellas la definición de áreas fluviales protegidas, desarrollo de proyectos de investigación acerca de su ecología y biología, programas de educación, y de planes de manejo que permitan su protección y aprovechamiento sostenible (Castro-Herrera, 2002; MoralesBetancourt et al., 2012a).

Finalmente, la percepción de los habitantes de la región sobre las tortugas, basada casi exclusivamente en su uso para consumo y comercio (éstas son consideradas un recurso de libre acceso), y la casi nula identificación de la función y valor ecológico de estas especies, establecen un reto para los manejadores de la vida silvestre en esta región, ya que será necesario adelantar programas de educación ambiental y cultural que propendan por el establecimiento de un vínculo sostenible entre las comunidades asentadas sobre la cuenca del río La Miel y las tortugas allí presentes. 


\section{AGRADECIMIENTOS}

A la Vicerrectoría de Investigaciones y Postgrados de la Universidad de Caldas. A la Doctora Vivian Páez (Universidad de Antioquia) por sus enseñanzas. A Verónica Duque por su apoyo en actividades de campo. A Ana María Jaramillo por su hospitalidad y a los pescadores de la zona, particularmente a Don Edgar Cupitra quien nos prestó apoyo logístico para el trabajo de campo. De manera especial agradecemos las sugerencias de dos revisores y del Comité Editorial, que mejoraron la presentación y alcances del estudio.

\section{POTENCIAL CONFLICTO DE INTERESES}

Los autores declaran que no existe conflicto de interés alguno por la realización y publicación de la presente investigación.

\section{FUENTES DE FINANCIACIÓN}

La Vicerrectoría de Investigaciones y Postgrados de la Universidad de Caldas financió la presente investigación en el marco del proyecto "Uso de hábitat del ensamble de tortugas dulceacuícolas en un tramo del río La Miel (Norcasia, Caldas)", Código 0552014.

\section{REFERENCIAS}

- Arroyave, F.J.; Romero, O.Y.; Bonilla, M.A. y Hurtado, R.G. (2014). Tráfico ilegal de tortugas continentales (Testudinata) en colombia: una aproximación desde el análisis de redes. Acta Biológica Colombiana, 19(3), 381-392.

- Bath, A.J. (1996). Increasing the applicability of human dimensions research to large predators. Journal of Wildlife Research, 1, 215-220. 
- Bennett, E.L. y Robinson, J.G. (2000). Hunting for sustainability: the start of a synthesis. En Robinson, J.G. y Bennett, E.L. (Eds.), Hunting for sustainability in tropical forest (pp. 499-519). USA: Columbia University Press.

- Cantillo-H., E.E. y Rangel-Ch., J.O. (2011). Estudio de caso aspectos de la estructura y dinámica de la sucesión vegetal en localidades del municipio de Norcasia, Caldas. En Rangel-Ch., J.O. (Ed.), Colombia diversidad biótica XI. Patrones de la estructura y de la riqueza de la vegetación en Colombia (pp. 127-144). Bogotá, D.C.: Universidad Nacional de Colombia.

- Castaño-Mora, O. (2002). Libro rojo de reptiles de Colombia. Bogotá, Colombia: Instituto de Ciencias Naturales - Universidad Nacional de Colombia, Ministerio de Medio Ambiente, Conservación Internacional - Colombia.

- Castro, A.; Merchán, M.; Garcés, M.; Cárdenas, M. y Gómez, F. (2013). Uso histórico y actual de las tortugas charapa (Podocnemis expansa) y terecay (Podocnemis unifilis) en la Orinoquia y la Amazonia. Biota Colombiana, 14(1), 4564.

- Castro-Herrera, F. (2002). Podocnemis lewyana. En Castaño-Mora, O. (Ed.), Libro rojo de reptiles de Colombia (pp. 92-94). Bogotá, Colombia: Instituto de Ciencias Naturales - Universidad Nacional de Colombia, Ministerio de Medio Ambiente, Conservación Internacional - Colombia.

- IUCN. (2014). The IUCN Red List of Threatened Species. http://www.iucnredlist.org

- Moll, D. y Moll, E.O. (2004). The ecology, exploitation and conservation of river turtles. New York: Oxford University Press.

- Morales-Betancourt, M.A.; Lasso, C.A.; Páez, V.P.; Trujillo, F.; Vargas-Ramírez, M.; Forero-Medina, G.; Hernández, O. y Trujillo, G. (2012a). Estrategias para la conservación de las tortugas continentales de Colombia. En Páez, V.P.; MoralesBetancourt, M.A.; Lasso, C.A.; Castaño-Mora, O. y Bock, B.C. (Eds.), Biología y conservación de las tortugas continentales de Colombia (pp. 495-521). Bogotá, D.C., Colombia: Instituto de Investigación de Recursos Biológicos Alexander von Humboldt (IAvH). 
- Morales-Betancourt, M.A.; Lasso, C.A.; Trujillo, F.; De la Ossa, J.; Forero, G. y Páez, V.P. (2012b). Amenazas a las poblaciones de tortugas continentales de Colombia. En Páez, V.P.; Morales-Betancourt, M.A.; Lasso, C.A.; Castaño-Mora, O. y Bock, B.C. (Eds.), Biología y conservación de las tortugas continentales de Colombia (pp. 453-492). Bogotá, D.C., Colombia: Instituto de Investigación de Recursos Biológicos Alexander von Humboldt (IAvH).

- Ojasti, J. y Dallmeier, F. (2000). Manejo de Fauna Silvestre Neotropical. Washington D.C.: Smithsonian Institution/MAB Biodiversity Program.

- Páez, V.P.; Morales-Betancourt, M.A.; Lasso, C.A.; Castaño-Mora, O. y Bock, B.C. (2012). Biología y conservación de las tortugas continentales de Colombia. Bogotá, D.C., Colombia: Instituto de Investigación de Recursos Biológicos Alexander von Humboldt (IAvH).

- Primack, R.; Rozzi, R.; Massardo, F. y Feinsinger, P. (2001). Conservación y desarrollo sustentable a niveles local y nacional. En Primack, R.; Rozzi, R.; Feinsinger, P.; Dirzo, R. y Massardo, F. (Eds.), Fundamentos de conservación biológica. Perspectivas latinoamericanas (pp. 585-618). México: Fondo de Cultura Económica.

- R Development Core Team. (2011). R: a language and environment for statistical computing. R Foundation for Statistical Computing.

- Racero-Casarrubia, J.A.; Vidal, C.C.; Ruiz, Ó.D. y Ballesteros, J. (2008). Percepción y patrones de uso de la fauna silvestre. Revista de Estudios Sociales, 31, 118-131.

- Rueda-Almonacid, J.V.; Carr, J.L.; Mittermeier, R.A.; Rodríguez-Mahecha, J.V.; Mast, R.B.; Vogt, R.C.; Rhodin, A.G.J.; Ossa-Velázquez, J.d.I.; Rueda, J.N. y Goettsch Mittermeier, C. (2007). Las Tortugas y Cocodrilianos de los Paises Andinos del Trópico. Bogotá, D.C.: Conservación Internacional - Editorial Panamericana, Formas e Impresos.

- Valdelomar, V.; Ramírez-Vargas, M.A.; Quesada-Acuña, S.G.; Arrieta, C.; Carranza, I.; Ruiz-Morales, G.; Espinoza-Bolaños, S.; Mena-Villalobos, J.M.; Brizuela, C.; Miranda-Fonseca, L.; Matarrita-Herrera, M.; González-Venegas, J.; Calderón-Sancho, E.; Araya, J.F.; Sauma-Rossi, Á.; Sandoval-Hernández, I. y Gómez-Lépiz, A. (2012). Percepción y conocimiento popular sobre el 
cocodrilo Crocodylus acutus (Reptilia: Crocodylidae) en zonas aledañas al río Tempisque, Guanacaste, Costa Rica. Cuadernos de Investigación UNED, 4(2), 191-202.

- Vallejo-Joyas, M.ı.; Londoño-Vega, A.C.; López-Camacho, R.; Galeano, R.; Álvarez-Dávila, E. y Devia-Álvarez, W. (2005). Establecimiento de parcelas permanentes en bosques de Colombia (Vol. 1). Bogotá D.C., Colombia: Instituto de Investigación de Recursos Biológicos Alexander von Humboldt.

- Van Dijk, P.P.; Iverson, J.B.; Rhodin, A.G.J.; Shaffer, H.B. y Bour, R. (2014). Turtles of the World, 7th Edition: Annotated Checklist of Taxonomy, Synonymy, Distribution with Maps, and Conservation Status. Chelonian Research Monographs, 5(7), 000.329-479.

1.Bióloga. Grupo de Investigación en Ecosistemas Tropicales, Facultad de Ciencias Exactas y Naturales, Universidad de Caldas. Manizales, Colombia.jennyza90@hotmail.com. ORCID: 0000-0001-9054-7533

2. Dr. en Ciencias mención Sistemática y Ecología. Docente, Departamento de Desarrollo Rural y Recursos Naturales, Facultad de Ciencias Agropecuarias, Universidad de Caldas; Grupo de Investigación en Genética, Biodiversidad y Manejo de Ecosistemas (GEBIOME). Manizales, Colombia. Facultad de Ciencias, Universidad del Tolima. Ibagué, Colombia. giovany.guevara@ucaldas.edu.co ggcolombia@gmail.com. ORCID: 0000-0002-2373-1805

3. Dr. en Ciencias mención Ecología y Biología Evolutiva. Docente, Departamento de Desarrollo Rural y Recursos Naturales, Facultad de Ciencias Agropecuarias, Universidad de Caldas; Grupo CERES (Centro de Estudios Rurales). Manizales, Colombia.gabriel.castano_v@ucaldas.edu.co. ORCID: 0000-0001-5327-8063

Para citar este artículo: Zapata-Ciro, J.; Guevara, G. y Castaño-Villa, G.J. (2016). Conocimiento popular y perspectivas de conservación sobre las tortugas continentales en la parte baja del río La Miel (Colombia). Revista Luna Azul, 43, 15-28. Recuperado de http://200.21.104.25/lunazul/index.php?option=com_content\&view=article\&id=191 
Esta obra está bajo una Licencia de Creative Commons Reconocimiento CC BY

(c) (i) 